\title{
Making it More Secure
}

\section{The Technical and Social Challenges of Expanding the Functionality of an Existing HPC Cluster to Meet University and Federal Data Security Requirements}

\author{
Jason Christopher ${ }^{\dagger}$ \\ Research IT \\ UC Berkeley \\ Berkeley, CA, USA \\ jchristo@berkeley.edu
}

\author{
Gary Jung \\ Scientific Computing Group \\ Lawrence Berkeley National Lab \\ Berkeley, CA, USA \\ gmjung@lbl.gov
}

\author{
Christopher Doane \\ Information Security and Policy \\ UC Berkeley \\ Berkeley, CA, USA \\ cdoane@berkeley.edu
}

\begin{abstract}
As High Performance Computing and Big Data analytics become more commonplace, we see researchers applying these tools in new areas. Indeed, in the past few years, we've seen the use of HPC in diverse areas such as archeology, public policy, and digital humanities. So it comes as no surprise that many life science researchers are now approaching us to use large scale computation and data analytics on their sensitive data sets, such as de-identified patient or genomics data, for the purposes of scientific inquiry. At UC Berkeley, this has become a pressing issue, as existing faculty need a place to do research on sensitive data. And we knew of at least one instance where it affected the campus' ability to recruit a new faculty member. We had a clear imperative for action!
\end{abstract}

An informal survey informed us that most other institutions built a new dedicated system to support their sensitive data research, including identified and HIPAA data. This paper is a case study of how we met this need by using a methodology to apply our campus cybersecurity framework, with the help of our institution's cybersecurity team, to convert our traditional production HPC cluster, with over 2000 users across 100 research groups, and our virtual machine service offering, to also support this type of research. Our efforts show that it is not only possible, but also that it is also a practical alternative to take this approach instead of building a new environment.

The field of information security focuses on defense-in-depth and as yet and offers no turnkey solutions that would prevent security incidents and breaches of data. As a result, the focus of most university and research lab information security groups is on preparing for detection of a breach after the fact and limiting its scope of impact. These realities combine such that to do secure computing in a high performance computing (HPC) cluster or on virtual machines in the Cloud, one must implement technical security controls, and write a host of process and audit documentation, which is both labor-intensive and on-going.

The paper describes our work at UC Berkeley to take an existing HPC cluster, with a base level of data security controls and procedures in place, and reconfigure it to meet more secure university and federal requirements, while maintaining the same computing experience and functionality on the system for users who are not computing over sensitive data. In other words, this is a study in configuring a hybrid HPC system for computing over non-sensitive and sensitive data alike, and our work to develop the policy and procedures to meet our information security requirements. It describes in detail the technical as well the educational and partner-building work we did at our institution to make this work a success.

Publication rights licensed to ACM. ACM acknowledges that this contribution was authored or co-authored by an employee, contractor or affiliate of the United States government. As such, the United States Government retains a nonexclusive, royaltyfree right to publish or reproduce this article, or to allow others to do so, for Government purposes only. PEARC '19, July 28-August 1, 2019, Chicago, IL, USA (C) 2019 Copyright is held by the owner/author(s). Publication rights licensed to ACM. ACM 978-1-4503-7227-5/19/07 ..\$15.00

https://doi.org/10.1145/3332186.3332250

\section{CCS CONCEPTS}

- Information systems $\sim$ Computing Platforms - Security and Privacy $\sim$ Systems Security

\section{KEYWORDS}

High Performance Computing, research computing, information security

\section{ACM Reference format:}

Jason Christopher, Gary Jung and Christopher Doane. 2019. Making it More Secure: The Technical and Social Challenges of Expanding the Functionality of an Existing HPC Cluster to Meet University and Federal Data Security Requirements. In Proceedings of Practice \& Experience in Advanced Research Computing conference (CHICAGO'19). Chicago, Ill, USA, 2 pages.

\section{Data Protection Level}

We anticipate the current demand, along with funding opportunities, for more secure computational environments will only increase. Our challenge was to meet requirements for computing over PL1 data (UC Berkeley defined) and NIH dbGaP data, where Protection Level 1 data are defined as:

Information intended for release only on a need-to-know basis, including personal information not otherwise classified 
as Level 0,2 or 3, and data protected or restricted by contract, grant, or other agreement terms and conditions, e.g.,:

- $\quad$ FERPA student records (including Student ID)

- $\quad$ staff and academic personnel records (including Employee ID)

- licensed software/software license keys

- library paid subscription electronic resources

NIH dbGap data are genotype and phenotype data that must be protected at roughly the same level of protection and with the same controls used for our institutional PL1 data. It should be noted that each data provider has its own requirements for data protection and these need to be carefully reviewed to ensure compliance.

\section{Our Approach}

As mentioned above, our approach to meeting researcher needs in this area was based on practicality, but we were also governed by: 1) expedience, 2) cost and 3) a desire to safeguard the experience of existing users of our HPC system. We were determined to not make changes on our systems -- add controls and processes -- that would adversely impact the experience of existing users. New faculty already on campus and other new hire faculty arriving the following year required some form of secure computing, underscoring our need for expedience.

Informal interview surveys of other institutions indicated that other schools hosted data at multiple protection levels (for us, PL1 and PL2) together in the same environment, and that a new HIPAA environment would take over a year to launch. Cost was an additional factor. We needed something cheaper than a de novo system. Leveraging the investment already made in our existing HPC system was then an obvious consideration we had to make.

Finally, while noting that some other institutions were hosting PL1 level environments without going through a process to ensure they were meeting all security requirements, such as working with their information security teams, we elected to engage the expertise of our information security and policy team immediately in the process. This partnership was instrumental to our ultimate success.

\section{Overview of Our Process}

To meet our security requirements and project goals, we had to complete a comprehensive system security plan, known at our local institution, UC Berkeley, as a Minimum Security Standard for Electronic Information (MSSEI) Self-Assessment Plan, or MSSEI Plan. This Plan includes not only technical, but also administrative and process controls.

The Plan is meant to capture the breadth and depth of the technical and process work needed to secure a system, with links out to additional supporting documents, such as an Incident Response Plan and Researcher Use Agreement. The process for completing the Plan is one of repeated draft by the system owner (us) and review by Information Security, until the majority of the configuration and documentation are understood and completed. Once the Plan is completed, the Information Security team signs off on the Plan, effectively certifying that system as meeting a particular level of security. The first sections of the Plan included a review of the architecture and workload, so that everyone had a common understanding of the system. This was the first HPC cluster that our ISP team had considered and they stated that it was the most complicated system they had encountered to date. A table of contents for the full Plan may be found below:

\begin{tabular}{|l|l|}
\hline Table of Contents for MSSEI & 7.1 No Core system on \\
Plan & Mobile/Wireless Devices \\
& 7.2 Data Encryption at Rest \\
Project System Overview & 7.3 Data Encryption in Transit \\
Target Audience & 8.1 Security and Privacy \\
Architectural Model & Training \\
Data Flow Description & 9.1 Separation of System \\
Support Model & Resources \\
& 10.1 Use of Admin Accounts \\
1.1 Covered System & on Secure Devices \\
Registration & 10.2 Admin Account Security \\
1.2 Registration Review & 11.1 Managed Hardware \\
2.1 Managed Software & Firewall \\
Inventory & 11.2 Protected Subnet \\
3.1 Secure Device & 12.1 Audit Logging \\
Configuration & 13.1 Controlled Access Based \\
4.1 Vulnerability Assessment & on Need to Know \\
and Remediation & 14.1 Account Monitoring and \\
4.2 Authenticated Scans & Management \\
4.3 Intrusion Detection & 15.1 Data Encryption in transit \\
5.1 Malware Defense & 15.2 Data Encryption on \\
5.2 Auto-Run Configuration & Removable Media \\
6.1 Secure Coding Practice & 15.3 Secure Deletion \\
6.2 Application Security & 15.4 Data Access Agreement \\
Testing & 16.1 Incident Response \\
6.3 Commercial Software & Planning \\
Assessment & 16.2 Incident Response Plan \\
& Availability \\
& 16.3 Incident Response \\
Training
\end{tabular}

Note: Some of the section listed above required minimal to no changes, while others required significant work.

It should also be noted that this project enjoyed a high level of support and optics from our Vice Chancellor of Research Office, multiple deans, faculty from disparate and related departments, as well as our campus CIO and Chief Academic Technology Officer. As a result, it was an "all hands on deck" project, and we hoped that a timely, on schedule, completion of the project would help us in our planned bid for additional funding the following fiscal year for a new security project. Likewise, delayed completion, could bear direct negative consequences on our intended requests. 


\section{The How and When of Encryption}

Early in the process, we made an assumption that we would be able to make the required changes to the HPC system without impacting our existing users, those who were not computing with sensitive data. We regarded this as absolutely essential, as we were unwilling to change the use model for this population. Since most of the work to meet our goal, we assumed, was process and documentation-based, we felt we had a very strong chance of doing this. However, as we began work on the Plan, the NIH $\mathrm{dbGaP}$ requirements posed some interesting challenges to our thinking, specifically the possible consideration of whether our HPC environment should be considered the same as a Cloud platform because they are similar in that they are both multitenant environments. If we did consider them the same, then a set of requirements having to do with encryption at rest would apply.

On one hand, in a very broad reading, the campus HPC cluster could be considered a cloud service by virtue of being multi-tenant. On the other hand, as a well-managed HPC cluster, it is firewalled/not on the public internet, not elastic, and access is controlled through account review, one-time passwords, and typical HPC scheduling abstractions. Although we were assuming that the intent of the cloud computing restrictions is to address the risks of using public and/or API accessible cloud infrastructure and not because of the multi-tenancy, our ISP team wanted a statement from NIH on the intent of the requirement.

Interestingly, we asked NIH for a clarification to which they responded with a reiteration of the policy in question. This was not helpful. In the end, we took a conservative approach and implemented a policy that required the data to be encrypted at rest in order to satisfy our ISP team. This was another example of how securing an HPC cluster was new to our ISP team and the questions about what controls should apply.

\section{Partnership and Educational Challenges}

The Research Computing and Information Security groups at our university are collegial partners, but historically the two groups have focused on different populations of users and different computing systems. Research computing is about clusters and more recently, Clouds. Information security has been more focused on administrative and enterprise computing. This is changing very rapidly, but in the context of our particular challenge here, it meant that the two groups would have to gain a better understanding of each other's work and focus before we could settle on a plan of action.

A very aggressive timeline for completion of this project injected a lot of pressure on both teams, and some initial tensions. But these tensions melted as we began to review the challenges together and a sense of co-ownership of the problem emerged.

Preliminary meetings between the two groups were focused on describing the architecture of our HPC system and how specific information requirements might be applied to such a system. This challenge got us off to a slow start, but ultimately turned into an opportunity and stimulus to completion. As knowledge and understanding of HPC architecture and security challenges grew in the minds of the project team members, a sort of co-ownership of the problem slowly emerged. Whereas at the outset, the information security team was perceived as the arbiters of security, they quickly came around to being perceived by the research computing team as essential, engaged and trusted partners -- both teams equally bent on solving the problem of how we could transform our existing HPC cluster to meet the all the security requirement, without changing its functionality for users who are not computing with sensitive data. In retrospect, it seems that this kind of coming together was predictable -- most technical people are at least mildly if not strongly curious about other technical systems -- and this was an opportunity for all project team members to expand their knowledge and do meaningful work together over time, a recipe which often correlates with success.

\section{Technical Challenges and Architectures}

To meet security requirements for hosting PL1 data, we had to change some minor aspects of network architecture. We eliminated dual-homed hosts, our visualization and JupyterHub nodes, so that everything except the DTN was behind the firewall, and installed a host-based firewall agent on the DTN. We also installed an Intrusion Detection System (IDS) on the DTN, to account for no IDS present on the Science DMZ.

Our Jupyterhub and viz nodes were straddling both a firewalled subnet and the public internet. In honesty, these devices were configured this way because we wanted to add them into production without having to reconfigure our firewall which was fragile and no longer supported by our network team. As part of the PL1 process, we upgraded to a new modern firewall and then moved the JupyterHub and Viz nodes behind it - thereby eliminating any secondary access to our private subnet except through the firewall and the Science DMZ

To meet the security requirements for our proposed system we had to use the Center for Internet Security Configuration Assessment Tool (CIS-CAT) on our system to determine possible vulnerabilities. Running the tool returns a spreadsheet report of tests with pass/fail results. There are more than 200 individual tests included in the tool. Initially, CIS-CAT returned a result in the $50 \%$ pass range but as we learned more about how to use the tool, we were able to achieve a result of $81 \%$ passed. Of the $19 \%$ of the tests that failed, many of them were not applicable to our cluster environment. Those that were applicable were mitigated by implementation of new technical configurations and/or process changes.

While we were required to use CIS-CAT by our information security team, we did not have to return a score of $100 \%$ to get our system approved. Our information security team wanted us to use the tool to get a better understanding of security issues in play, to address potential vulnerabilities intentionally, and to document both our claims of "not applicable" and our mitigation strategies for items that were applicable. In the end, CIS-CAT provided us with a security baseline, that we could revisit and re-run as the system evolves over time. Having a standardized test allows us to be more intentional about security strategies and the process we 
have built around using it will undoubtedly make our system more secure.

Our HPC system is fairly standard, it follows a Beowulf architectural model used for building Linux clusters. However as a research computing cluster, it does pose unique challenges that are different from enterprise environments. A research cluster, by design, is supposed to allow researchers to run a myriad of software from collaborators, other institutions, or home grown. We also have to deal with a multi-tenant shared environment. Lastly, technology such as the Lustre parallel filesystem or infiniband may be foreign to cybersecurity experts used to working on enterprise servers.

In addition to use of CIS-CAT to help us refine the configuration of our nodes, we also made some architectural changes -- mostly around moving some nodes to a location behind the firewall and securing our Data Transfer Node with a host-based intrusion detection system.

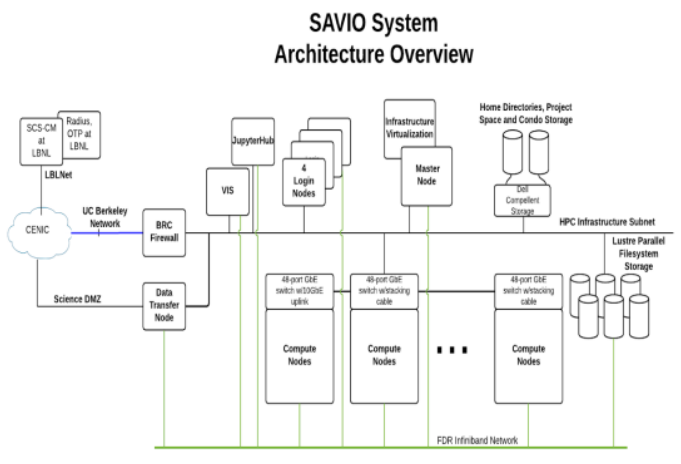

The SAVIO System, our HPC cluster, follows the standard Beowulf architecture used for building Linux clusters, in that it is a single system built from discrete PCs.

\section{Researchers, Roles and Responsibilities}

In addition to the partnership and technical challenges described above, a significant amount of work went into developing roles, responsibilities and processes for all parties, including system providers, campus information security staff, and researchers. We developed a shared responsibility model for use of the system, and documented the model in an MOU or Researcher Use Agreement. Our approach to the agreement was to take responsibility for the security of the infrastructure, while the researchers, and for the researchers to take responsibility for managing the data. We are using policy controls instead of technical controls to secure the data.
Review and practice of new processes were entered into a shared security calendar (Google calendar layer). This was a simple but effective way to document and share our plans and the timely completion of work.

In addition to these document tools, we created a Cyber Security Incident Response Plan and a regular process by which we would familiarize researchers and systems administrators with their respective responsibilities. We also registered all nodes in the cluster with our campus Network Registration (NetReg) Service. This service identifies security contacts for systems that compute over sensitive data and the IP address space used by the system so that in the event of a possible breach, information security staff can more quickly determine scope and impact of the event.

The work described in this section has led to less system administrator time devoted to policing the system.

\section{Conclusions}

This paper describes the challenges and solution to modifying an existing HPC cluster to support compute over sensitive date. By use of a combination of technical controls, planned process for both researcher use and system administration, documentation and a shared responsibility model for security between service provider (us) and researchers, we were able to create a new, more secure HC cluster computing option for our researchers.

Completion of this work has been very well-received and has had a multiplier effect. Accolades from researchers, deans of departments and senior campus IT management were received within minutes of a late Friday afternoon announcement of launch. Success on this project has accelerated our work to raise our virtualized analytics environments to the PL1 level. With both HPC and on-prem Cloud-based virtual machines at the PL1 level of operation, we have a comprehensive set of offerings for researchers.

\section{Looking Ahead}

The partnership that emerged between our research computing group and information security teams during this project enabled us to be successful, and set the foundation for future partnership to build an additional, HIPAA-compliant secure computing environment in the coming year. This environment will provide both HPC and very large virtual machines to support researchers from a breadth of disciplines.

Our campus as a whole is moving toward a more a secure, and documented approach to information security. The kind of security plan we had to complete for our project will soon have to 
be completed by every department on campus. Within our own larger organization - Research, Teaching and Learning - other architecture and developer groups are also working on similar security plans for their projects and services that require compute over sensitive data. In light of this, groups in our organization have agreed to share notes, templates and best practices for creating a security plans. This outcome should have the positive effect of making all individuals and teams more aware of information security best practices, as well as identifying specific know architectures that pass muster. These architectures could then be shared more broadly with the wider campus community, and perhaps even with other institutions both within the University of California system and with external institutions.

\section{REFERENCES}

[1] NIH Security Best Practices for Controlled-Access Data Subject to the NIH Genomic Data Sharing Policy. March 9, 2015. https://osp.nih.gov/wpcontent/uploads/NIH_Best Practices for Controlled-Access to the Data Subject to the NIH GDS Policy.pdf.

[2] UC Berkeley / Information Security and Policy - A-Z Policy Catalog: Laws and Regulations. See: https://security.berkeley.edu/policy/laws-regulations.

[3] UC Berkeley / Information Security and Policy - Minimum Security Standard for Electronic Information. July 2013. See: https://security.berkeley.edu/minimum-security-standard-electronicinformation-effective-july-2013

[4] Protecting Controlled Unclassified Information in Nonfederal Systems and Organizations. June 6, $2018 . \quad$ See: https://csrc.nist.gov/publications/detail/sp/800-171/rev-1/final

[5] NIH Security Best Practices for Controlled-Access Data Subject to the NIH Genomic Data Sharing (GDS) Policy. See: https://osp.od.nih.gov/wpcontent/uploads/NIH Best Practices for Controlled-Access Data Subject to the NIH GDS Policy.pdf 